\title{
Modulatory effect of high molecular weight polyethylene glycols on drug release from ibuprofen matrix tablets
}

\author{
*ERAGA, SO; EBOH, E; IWUAGWU, MA \\ Department of Pharmaceutics and Pharmaceutical Technology, \\ Faculty of Pharmacy, University of Benin, Benin City 300001, Nigeria \\ *Corresponding author: Eraga, Sylvester $O$. \\ E-mail: eragaso@uniben.eduTel.: +234-8030884928
}

\begin{abstract}
The work aim at investigating the channeling or modulatory effects of polyethylene glycol (PEG) (MW 4000 and 6000) on drug release from ibuprofen sustained release formulation. Different batches of ibuprofen matrix granules and tablet were prepared by melt granulation using different concentrations of carnauba wax and PEG at different ratios. The granule flow properties and various tablets parameters were evaluated using standard procedures. Drug release kinetics and mechanisms from the tablets were investigated as well as DSC and FTIR drug-excipients compatibility. The granules showed increasingly close packing with increase in the amounts of PEG incorporated. All the tablets did not meet compendial specifications with regard to crushing strength and friability. The release rate and extent of release were found to be influenced by the amount of PEG used as well as the carnauba wax concentration. PEG combination of equal amounts produced the highest release of $91 \%$ in the formulation prepared with $12.20 \% \mathrm{w} / \mathrm{w}$ of carnauba wax while a 1:2 combination in tablets prepared with $24.40 \% \mathrm{w} / \mathrm{w}$ of carnauba wax gave a maximum drug release of $83 \%$. Drug release kinetic and mechanism were most consistent with the Higuchi model hence the release was diffusion mediated. DSC and FTIR studies showed no interactions between ibuprofen and the excipients. Carnauba wax-PEG system can be used successfully as a matrix former to sustain the release of ibuprofen for over $6 \mathrm{~h}$. The studies indicate that the proper balance between a matrix former and a channeling agent can produce a desired drug dissolution profile. (C) JASEM

https://dx.doi.org/10.4314/jasem.v21i5.9
\end{abstract}

Keywords: carnauba wax, PEG, granulation, release modifier, ibuprofen, tablets

The need to improve dosage convenience and also enhance patient's compliance has led to the search for various ways of modifying drug release from solid dosage forms using polymers such as waxes, lipids and polyethylene glycols (PEGs). Among these polymers, PEG has emerged as the most favourable carriers because of its versatility. It is non-toxic, nonimmunogenic, non-antigenic, soluble in water and FDA approved (Veronese and Pasut, 2005). A variety of PEGs, both linear and branched, with different molecular weights have been exploited successfully as drug carriers to achieve controlled release (Maheshwari et al., 2003), mask taste, (Robson, 1999) and improve stability (Paradkar et al., 2004).

Carnauba wax is a natural wax used as an ingredient in many pharmaceutical formulations for its hypoallergenic and emollient properties. Many carnauba wax-based lipophilic matrix tablets have been successfully designed to protect drugs from hydrolytic degradation (Avbunudiogba et al., 2013) and control or sustain drug release (Ahmed and Khalil, 2014; Arhewoh et al., 2015; Onyechi and Okafo, 2016).

In the use of carnauba wax as a matrix former in the sustained release of drugs, being a solid wax, drug release from its porous matrix structure is controlled by the penetration of fluid into the porous matrix to dissolve the incorporated drug, followed by diffusion of the dissolved molecules out of the matrix. Hence, liquid penetration into the matrix is the rate limiting step in the dissolution and release of drug from the system. The use of agents, known as channeling agents or rate modifiers, capable of affecting the rate limiting step by modifying the processes of matrix liquid penetration and drug diffusion from the matrix is currently being explored (Uddin et al., 2009; IbnRazzak et al., 2009; Ahmed and Khalil, 2014). Agents such as mannitol, starch, lactose, PEG 4000, PEG 6000, PVP K30, sodium chloride, microcrystalline cellulose and dicalcium phosphate have been successfully used as channeling agents in sustained release formulations (Uddin et al., 2009; Ibn-Razzak et al., 2009; Ahmed and Khalil, 2014; Bruschi, 2015).

Several techniques have been employed in the formulation of carnauba wax matrix controlledrelease granules and tablets, which includes: melt coating, melt granulation, melt extrusion and direct compression. The application of these techniques depends on the physicochemical properties of the drug as well as the desired properties of the final product.

Ibuprofen is a non-steroidal anti-inflammatory agent and it belongs to class II (High permeability low solubility) of the Biopharmaceutical Classification System (BCS). Its bioavailability is limited by its solvation rate. A correlation between the in vivo bioavailability and the in vitro solvation can be found. Ibuprofen is a suitable candidate for sustained drug release delivery system due to its short half-life necessitating high frequency of administration. 
The main objective of this work is the development and optimization of a sustained release formulation of ibuprofen using carnauba wax (a hydrophobic polymer) as the matrix former and to evaluate the effects of two hydrophilic polymers (PEG 4000 and PEG 6000) of varying molecular weights as channeling agents on ibuprofen release from the matrix tablet.

\section{MATERIALS AND METHOD}

Materials; Ibuprofen powder and microcrystalline cellulose were gift samples from Edo Pharmaceuticals Ltd, Benin City, Nigeria. Polyethylene glycols (Mol.Wt 6000 and 4000) were purchased from Sigma-Aldrich, Germany. Magnesium stearate and talc (BDH Chemicals, UK), potassium hydrogen phosphate and sodium hydroxide (Merck, Germany). Carnauba wax was obtained from the laboratory stock in the Department of Pharmaceutics and Pharmaceutical Technology, Faculty of Pharmacy, University of Benin, Benin City, Edo State, Nigeria.
Methods, Granulation; Using the formula in Table 1, melt granulation method was used in formulating twelve batches (A-L) of the ibuprofen granules that were compressed into tablets by direct compression. For each batch, the required quantity of carnauba wax sufficient to produce 100 tablets was melted in a stainless steel vessel over a hot water bath $\left(>90{ }^{\circ} \mathrm{C}\right)$ with continuous stirring. The specific amounts of ibuprofen and microcrystalline cellulose (MCC) were added to the melted wax with continuous stirring until a homogeneous mixture was produced. Thereafter, the amounts equivalent to the various ratios of PEG 4000 and 6000 were added into the molten mass and stirred continuously to form a homogeneous molten mass that was allowed to cool at room temperature. The cooled mass was pressed through a sieve of aperture size $710 \mu \mathrm{m}$ to give granules. The granules were evaluated for their flow properties and compressed into tablets using a single punch tableting machine (Manesty Machines, UK) after the geometric addition of the required amounts of magnesium stearate and talc and mixing intimately.

Table 1: Formula for the preparation of ibuprofen granules and tablets

\begin{tabular}{|c|c|c|c|c|c|c|c|c|c|c|c|c|}
\hline \multirow{2}{*}{$\begin{array}{l}\text { Ingredients } \\
\text { (mg) }\end{array}$} & \multicolumn{12}{|c|}{ Batches } \\
\hline & $\mathbf{A}$ & B & $\mathbf{C}$ & D & $\mathbf{E}$ & $\mathbf{F}$ & $\mathbf{G}$ & $\mathbf{H}$ & I & $\mathbf{J}$ & $\mathbf{K}$ & $\mathbf{L}$ \\
\hline Ibuprofen & 200 & 200 & 200 & 200 & 200 & 200 & 200 & 200 & 200 & 200 & 200 & 200 \\
\hline MCC & 150 & 140 & 140 & 130 & 120 & 120 & 150 & 140 & 140 & 130 & 120 & 120 \\
\hline Carnauba wax & 50 & 50 & 50 & 50 & 50 & 50 & 100 & 100 & 100 & 100 & 100 & 100 \\
\hline PEG 4000 & - & 10 & - & 10 & 10 & 20 & - & 10 & - & 10 & 10 & 20 \\
\hline PEG 6000 & - & - & 10 & 10 & 20 & 10 & - & - & 10 & 10 & 20 & 10 \\
\hline Magnesium stearate & 5 & 5 & 5 & 5 & 5 & 5 & 5 & 5 & 5 & 5 & 5 & 5 \\
\hline Talc & 5 & 5 & 5 & 5 & 5 & 5 & 5 & 5 & 5 & 5 & 5 & 5 \\
\hline
\end{tabular}

Drug-excipient interaction studies; DSC and FTIR compatibility studies were carried out on pure ibuprofen powder and the formulated granules to investigate any interaction between the drug and the excipients.

The DSC analysis was carried out using a Netzsch DSC 204F1 Phoenix apparatus (Netzsch Germany). Four milligrams of the sample was weighed into an aluminium pan and sealed. The seal was pierced and calibration of the calorimeter was carried out with indium. Heating of the sample was carried out at the rate of $10{ }^{\circ} \mathrm{C}$ per min from 30 to $350{ }^{\circ} \mathrm{C}$ under nitrogen at a flow rate of $70 \mathrm{ml} / \mathrm{min}$. FTIR analysis of the sample was carried out using FTIR-4100 Spectrophotometer (Shimadzu Co. Japan). The potassium bromide $(\mathrm{KBr})$ tablet method was used; five milligrams of the sample was blended with $\mathrm{KBr}$ to $200 \mathrm{mg}$. The powder was compressed using a Sigma KBr press into a tablet shape. The tablet was placed in the sample compartment and scanned at a range of $4000-750 \mathrm{~cm}^{-1}$.

Granule evaluations, Bulk density; Ten grams quantity of granules was poured gently into a $100 \mathrm{ml}$ measuring cylinder and the volume occupied by the sample without tapping was determined. The bulk density, which is the ratio of the weight of the granules and the volume of the granules, was determined using Equation 1.

Bulk density $=\frac{\text { Weight of granules }}{\text { Volume of granules }}$

Tapped density; The measuring cylinder containing the $10 \mathrm{~g}$ granules was tapped mechanically on a flat surface for about a 100 times to a constant volume which was recorded as the tapped volume. The tapped density that is the ratio of the weight of the granules and the tapped volume of the granules was determined using the Equation 2.

Tapped density $=\frac{\text { Weight of granules }}{\text { Tapped volume of granules }} \ldots$

Carr's consolidation index; Carr's index is the difference between the tapped and bulk density of the granules divided by the tapped density and the ratio 
expressed as a percentage. The indices of the various batches of granules were calculated using Equation 3.

Carr's index $=\frac{\text { Tapped density }- \text { Bulkdensity }}{\text { Tapped density }} \times 100 .$. (3)

Hausner's ratio; The ratio of the tapped density to the bulk density of the granules was calculated as the Hausner's ratio or quotient with Equation 4.

Hausner's ratio $=\frac{\text { Tapped density }}{\text { Bulk density }} \ldots \ldots .$.

Angle of repose; The angle of repose was measured according to the fixed funnel and free standing cone method (Richard, 1972). A funnel was clamped to the retort stand with its tip above a white plain paper placed on a flat horizontal surface. Granules equivalent to $10 \mathrm{~g}$ was carefully poured through the funnel while the efflux was closed and then opened after the granules have been poured completely. The granules flowed freely forming a cone. The mean diameters of the base of the granules cone and also the height of the granules were determined with the aid of a meter rule and the tangent of the angle of repose $(\theta)$ was calculated using Equation 5.

$$
\theta=\tan ^{-1} \frac{h}{r} \quad \ldots \ldots . .
$$

Where $h$ is the height of the heap of granules and $r$ is the radius of the circular base

Flow rate; The funnel method of Carstensen and Chan (1977) was employed. A funnel was clamped to the retort stand and a clean paper positioned directly at the base of the funnel efflux. Ten grams of the granule was introduced into the funnel with the efflux tube closed. The efflux was opened and the granules allowed to fall freely under gravity. The flow rate was calculated using Equation 6.

Flow rate $=\frac{\text { Weight of granules }}{\text { Time of flow }} \quad \ldots \ldots$.

Tablet evaluations, Weight uniformity and dimensions; The weight of each of 20 tablets was determined from each batch using an electronic balance while the thickness and diameter of each of ten tablets per batch were measured using a micrometre screw gauge (Gallenkamp). Their mean values and standard deviations were computed and recorded.
Crushing strength; Using a motorized tablet hardness tester (Campbell Electronics, Model HT-30/50, India), the crushing strength of ten individual tablets per batch was determined by diametric compression. The mean and standard deviation values were calculated and recorded.

Friability; Twenty tablets selected randomly from each batch were weighed and placed in the drum of a friabilator (Erweka GmbH, Germany) set to revolve at $25 \mathrm{rpm}$ for $4 \mathrm{~min}$. At the end, the tablets were brought out, de-dusted and re-weighed. Using Equation 7, the percentage loss in weight of the tablets was calculated and used as a measure of the tablet's friability.

$$
\text { Friability }=\frac{\text { Initial weight }- \text { Final weight }}{\text { Initial weight }} \times 100 \ldots
$$

Dissolution studies; Dissolution profiles of 6 randomly selected tablets per batch were determined using the BP basket method. The dissolution apparatus (Caleva ST7, UK) with a basket revolution of $50 \mathrm{rpm}$ and containing $900 \mathrm{ml}$ of $0.1 \mathrm{~N} \mathrm{HCl}$ solution thermostated at $37 \pm 0.5^{\circ} \mathrm{C}$ was used for the first $2 \mathrm{~h}$ and thereafter replaced with $900 \mathrm{ml}$ of phosphate buffer $\mathrm{pH} 7.2$ for the remaining $4 \mathrm{~h}$. Aliquots of $5 \mathrm{ml}$ were withdrawn at various time intervals dissolution fluid and replaced with an equivalent volume of the corresponding dissolution medium maintained at $37 \pm 0.5{ }^{\circ} \mathrm{C}$. The withdraw samples were then filtered (Whatman No. 1), diluted appropriately with the dissolution medium and analysed spectrophotometrically at wavelength of 221 $\mathrm{nm}$ (T70, PG Instruments Ltd). The percentage of drug released at each time interval was then calculated from the equation $(y=0.014 x+0.097)$ obtained earlier from the ibuprofen calibration curve.

Release kinetics; Data from the dissolution studies were fitted into various kinetic equations to determine the model and mechanism of ibuprofen release from the matrix tablets. The correlation coefficient $\left(r^{2}\right)$ for each rate order was calculated. The dissolution profile was considered to follow a particular rate order if the $\mathrm{r}^{2}$ value was $\geq 0.95$.

Zero-order: This was to determine the existence of a linear relationship between ibuprofen releasefrom the matrix tablet and time i.e., drug release rate is independent of concentration. The following equation was used to describe the release kinetic.

$\mathrm{Q}=\mathrm{kt}$

Where, Q is the cumulative amount of ibuprofen released at time $\mathrm{t}$ and $\mathrm{k}$ is the release rate constant. A 
graphical plot of cumulative amount of ibuprofen released versus time will be linear if the release obeys zero order release kinetics.

First-order: Wagner in 1969 proposed a first order drug release kinetic that described a concentration dependent drug release rate (Wagner, 1969). The following equation was used to describe the release kinetic.

$$
\text { In }(1-\mathrm{Q})=-\mathrm{kt} \quad \ldots \ldots \ldots \ldots
$$

Where, $\mathrm{Q}$ is the cumulative amount of ibuprofen released at time $\mathrm{t}$ and $\mathrm{k}$ is the release rate constant. A graphical plot of logarithm of amount of ibuprofen unreleased versus time will be linear if the release obeys first order release kinetics.

Higuchi model: This model was developed by Higuchi to describe the release of drug from an insoluble matrix as the square root of a timedependent process based on Fickian diffusion (Higuchi, 1968). It defines a linear dependence of the amount of drug released per unit of surface on the square root of time and it is expressed by the equation:

$\mathrm{Q}=\mathrm{kt}^{1 / 2}$

Where, Q is the cumulative amount of ibuprofen released at time $\mathrm{t}$ and $\mathrm{k}$ is the release rate constant. A graphical plot of cumulative amount of ibuprofen released against square root of time will be linear if the release obeys Higuchi equation.

Korsemeyer-Peppas model: Korsmeyer and coworkers in 1983 derived an empirical equation that described both Fickian and non-Fickian release of drug from polymeric delivery systems (Korsemeyer et al., 1983). The logarithm of the equation can be representing thus;

$\log \mathrm{Q}=\log \mathrm{k}+\mathrm{n} \log \mathrm{t}$

Where, Q is the cumulative amount of ibuprofen released at time $\mathrm{t}, \mathrm{k}$ is the release rate constant and $\mathrm{n}$ is the diffusional exponent determining the mechanism of transport of ibuprofen through the polymer matrix (0.45 (Fickian diffusion), $0.45<\mathrm{n}<$ 0.89 (Anomalous non-Fickian diffusion), 0.89 (CaseII transport) $>0.89$ (Super Case-II transport)). A plot of the logarithm of cumulative amount of ibuprofen released against logarithm of time will be linear if the release obeys Korsemeyer-Peppas equation.

Statistical analysis; The mean values of results obtained from the granule and tablet's evaluations were subjected to student's t-test at $5 \%$ level of significance using GraphPad InStat 3.10.

\section{RESULTS AND DISCUSSION}

Flow properties of granules; Results from the evaluation of the granule flow properties are shown in Table 2. The values of the bulk and tapped densities of the various batches of the granules in conjunction with their angles of repose values showed that the granules had increasingly close packing with increase in the amounts of PEG incorporated. The angle of repose values ranged from $24.80-34.80^{\circ}$ indicating granules with good to fair flow properties (Mehta and Barker, 1994).

Table 2: Micromeritic properties of the ibuprofen granules

\begin{tabular}{cccccc}
\hline Batch & $\begin{array}{c}\text { Bulk density } \\
\left(\mathbf{g} / \mathbf{c m}^{\mathbf{3}}\right)\end{array}$ & $\begin{array}{c}\text { Tapped density } \\
\left(\mathbf{g} / \mathbf{c m}^{\mathbf{3}}\right)\end{array}$ & $\begin{array}{c}\text { Angle of repose } \\
(\mathbf{o})\end{array}$ & $\begin{array}{c}\text { Carr's index } \\
(\boldsymbol{\%})\end{array}$ & $\begin{array}{c}\text { Hausner's } \\
\text { ratio }\end{array}$ \\
\hline A & $0.514(0.011)$ & $0.606(0.010)$ & $24.80(0.21)$ & $15.18(0.12)$ & $1.179(0.014)$ \\
B & $0.496(0.012)$ & $0.583(0.020)$ & $28.20(0.30)$ & $14.92(0.14)$ & $1.175(0.020)$ \\
C & $0.499(0.014)$ & $0.617(0.011)$ & $33.00(0.10)$ & $19.13(0.82)$ & $1.236(0.024)$ \\
D & $0.525(0.012)$ & $0.612(0.012)$ & $34.80(0.20)$ & $14.20(0.62)$ & $1.166(0.042)$ \\
E & $0.490(0.022)$ & $0.570(0.016)$ & $28.80(0.15)$ & $14.04(0.50)$ & $1.160(0.030)$ \\
F & $0.490(0.042)$ & $0.574(0.019)$ & $32.30(0.30)$ & $14.60(0.30)$ & $1.171(0.010)$ \\
G & $0.496(0.023)$ & $0.571(0.022)$ & $25.70(0.25)$ & $13.13(0.27)$ & $1.151(0.011)$ \\
H & $0.495(0.032)$ & $0.581(0.045)$ & $26.60(0.40)$ & $14.80(0.10)$ & $1.174(0.026)$ \\
I & $0.501(0.010)$ & $0.580(0.014)$ & $29.30(0.12)$ & $13.62(0.32)$ & $1.158(0.031)$ \\
J & $0.513(0.011)$ & $0.601(0.012)$ & $29.80(0.05)$ & $14.60(0.20)$ & $1.171(0.021)$ \\
K & $0.511(0.022)$ & $0.613(0.010)$ & $31.90(0.22)$ & $16.64(0.16)$ & $1.200(0.082)$ \\
L & $0.526(0.012)$ & $0.614(0.010)$ & $27.20(0.28)$ & $14.33(0.24)$ & $1.167(0.022)$ \\
\hline
\end{tabular}

*Standard deviation in parenthesis

Batches $\mathrm{A}$ and $\mathrm{G}$ granules containing only carnauba wax gave the lowest angle of repose values and they exhibited excellent flow properties. The compressibility indices and Hausner's ratios of the various batches of the granules which ranged from $13.13-19.13 \%$ and $1.151-1.236$ respectively, also showed the decrease in granule flowability with increasing concentrations of PEG. The decrease in granule flow with the incorporation of PEG may be attributable to the generation of finer granule particles by PEG due to incomplete mixing in the granulation process as a result of their different chemical nature. 
If this was the case, the higher the amounts of PEG, the higher the amounts of fines produced. These finer particles will tend to fill void spaces in-between the larger sized granules leading to more cohesive granules with poor flow properties (Eraga et al., 2014).

Drug-excipient compatibility; DSC thermogram of pure ibuprofen powder (Figure 1 (a)) exhibited a sharp endothermic trough at $78{ }^{\circ} \mathrm{C}$ corresponding to the melting point of ibuprofen and an indication of its purity and crystallinity while the thermogram of the granules (Figure 1 (b)) also showed the characteristic sharp endothermic trough of pure ibuprofen. This observation confirms the presence of ibuprofen in its characteristic physical and chemical form in the granules and rules out any possible interactions between ibuprofen and the excipients used in the formulation of the matrix tablets.

All the formulated tablets in the various batches passed the weight uniformity test, as the variation of no two tablets out of the total of 20 tablets used for the test fell outside $\pm 5 \%$ and none was outside \pm 10 $\%$ deviation from the average tablet weight (BP, 2009). The crushing strength values of the tablets indicated tablets of acceptable hardness except Batch $\mathrm{F}$ tablets, as values $\geq 4 \mathrm{kp}$ have been reported as optimal for acceptable tablet hardness (Rudnic and Schwartz, 2006). Batches A and G tablets containing only carnauba wax without PEG showed the highest crushing strength values of 11.75 and $12.75 \mathrm{kp}$, respectively, while the values of the other batches decreased with the incorporation of PEG and with increasing amounts of PEG. Also, the friability values of the tablets followed a reverse but similar trend as the tablet's crushing strength values with the least values being exhibited by Batches $A$ and $G$ tablets and an increase in friability with the incorporation of PEG and with increasing amounts of PEG. Batches E, $\mathrm{F}$ and $\mathrm{L}$ tablets did not meet the official compendial requirement of a $0.8-1.0 \%$ maximum loss of tested tablet weight. The decrease in tablet's hardness and the inverse correlation existing here between the tablet's hardness and friability may be as a result of the fines produced by PEG as earlier stated under granule properties. The filling of voids between particles by these finer particles might interfere with interparticle bond formation in the tablets leading to low interparticulate bridges and decreased area of contacts between particles and consequently conferring less resistance to tablet fracture and abrasion. Some authors have supported this indirect correlation between the hardness and friability of tablets. They have postulated that, depending on the fines filling these voids, they will either contribute towards the tablet hardness with lesser friability by facilitating solid interparticle bonds or lower the hardness with a resultant higher friability by weakening the particle bonds (Iwuagwu et al., 1986) (Oyi et al., 2009).

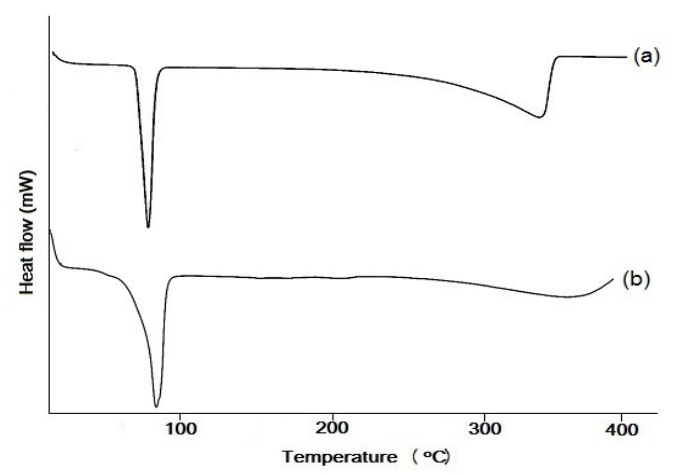

Fig 1: DSC thermograms of ibuprofen powder (a) and the prepared tablet granules (b).

The FTIR spectrum of pure ibuprofen (Figure 2 (a)) powder exhibited characteristic absorption bands at $1228.58,1422.61,1717.58$ and $2946.00 \mathrm{~cm}^{-1}$. These bands observed for ibuprofen remained unchanged when compared with the spectral data of the tablet granules (Figure 2 (b)). This observation ruled out the possibility of chemical interaction and complex formation between ibuprofen and the other tablet excipients during granulation.

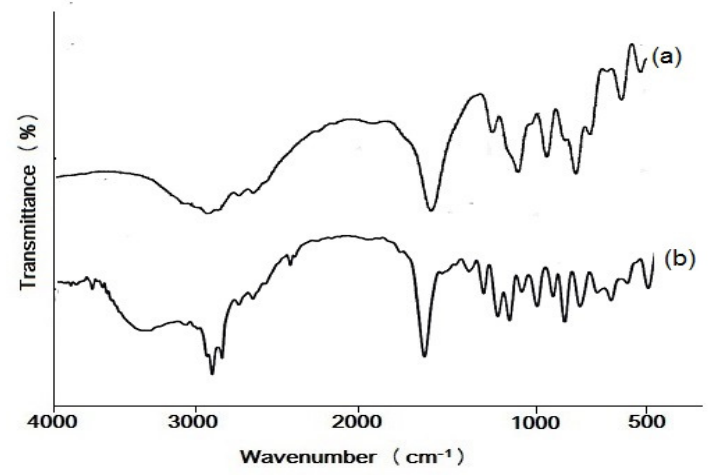

Fig 2: FTIR spectra of pure ibuprofen powder (a) and the prepared tablet granules $(\mathrm{b}$

Tablet properties; Results from the post-compression evaluations of the different batches of the formulated ibuprofen matrix tablets are outlined in Table 3. 
Table 3: Post compression parameters of the ibuprofen matrix tablet

\begin{tabular}{cccccc}
\hline \multirow{2}{*}{ Batch } & $\begin{array}{c}\text { Tablet } \\
\text { weight } \\
(\mathbf{m g})\end{array}$ & \multicolumn{2}{c}{ Tablet dimensions $(\mathbf{m m})$} & $\begin{array}{c}\text { Crushing } \\
\text { strength } \\
(\mathbf{k p})\end{array}$ & $\begin{array}{c}\text { Friability } \\
(\%)\end{array}$ \\
\cline { 3 - 4 } & & Thickness & Diameter & $11.75(0.18)$ & $0.50(0.03)$ \\
A & $410(0.03)$ & $2.98(0.01)$ & $12.63(0.02)$ & $7.63(0.11)$ & $0.86(0.01)$ \\
B & $405(0.04)$ & $2.96(0.04)$ & $12.64(0.03)$ & $5.50(0.18)$ & $0.87(0.02)$ \\
C & $410(0.02)$ & $2.98(0.03)$ & $12.62(0.01)$ & $5.37(0.30)$ & $0.95(0.01)$ \\
D & $408(0.02)$ & $2.99(0.03)$ & $12.63(0.01)$ & $4.25(0.10)$ & $1.03(0.04)$ \\
E & $410(0.03)$ & $2.96(0.02)$ & $12.62(0.03)$ & $3.80(0.20)$ & $1.10(0.03)$ \\
F & $410(0.02)$ & $2.97(0.02)$ & $12.63(0.02)$ & $12.75(0.17)$ & $0.46(0.03)$ \\
G & $412(0.03)$ & $2.98(0.04)$ & $12.64(0.03)$ & $7.63(0.13)$ & $0.73(0.02)$ \\
H & $412(0.02)$ & $2.99(0.04)$ & $12.63(0.01)$ & $7.25(0.25)$ & $0.82(0.02)$ \\
I & $412(0.03)$ & $2.26(0.03)$ & $12.62(0.03)$ & $5.00(0.45)$ & $0.84(0.02)$ \\
J & $405(0.02)$ & $2.98(0.03)$ & $12.63(0.03)$ & $4.87(0.21)$ & $0.99(0.03)$ \\
K & $408(0.02)$ & $2.97(0.03)$ & $12.62(0.03)$ & $4.00(0.14)$ & $1.04(0.02)$ \\
L & $408(0.02)$ & $2.99(0.03)$ & $12.64(0.02)$ & & \\
\hline
\end{tabular}

*Standard deviation in parenthesis

Table 4: Correlation coefficient $\left(\mathrm{R}^{2}\right)$ of the dissolution studies

\begin{tabular}{ccccc}
\hline \multirow{2}{*}{ Batch } & \multicolumn{3}{c}{ Correlation coefficient $\left(\mathbf{R}^{\mathbf{2}}\right)$} \\
\cline { 2 - 4 } & Zero order & First order & Higuchi & Korsmeyer-Peppas (n) \\
\hline A & 0.7429 & 0.8699 & 0.9186 & $0.9389(1.63)$ \\
B & 0.7646 & 0.9192 & 0.9347 & $0.9772(1.23)$ \\
C & 0.7842 & 0.9171 & 0.9451 & $0.9781(1.68)$ \\
D & 0.8958 & 0.9586 & 0.9794 & $0.9875(1.67)$ \\
E & 0.8648 & 0.9627 & 0.9751 & $0.9940(1.67)$ \\
F & 0.7965 & 0.9458 & 0.9505 & $0.9847(1.72)$ \\
G & 0.6296 & 0.8524 & 0.8151 & $0.8213(1.59)$ \\
H & 0.9030 & 0.9085 & 0.9804 & $0.9869(1.64)$ \\
I & 0.7513 & 0.9520 & 0.9225 & $0.9682(1.70)$ \\
J & 0.8040 & 0.9286 & 0.9285 & $0.9344(1.65)$ \\
K & 0.8296 & 0.9454 & 0.9623 & $0.9693(1.66)$ \\
L & 0.8100 & 0.9390 & 0.9361 & $0.9425(1.67)$ \\
\hline
\end{tabular}

Dissolution profile; The drug release profiles of the various batches of the ibuprofen matrix tablets are presented in Figures 3 and 4. Batches A and G tablets containing carnauba wax alone exhibited the highest ibuprofen release retardation with a maximum percentage drug release of 71 and $60.5 \%$ within $6 \mathrm{~h}$. The other batches of tablets, containing PEG showed variable percentage drug release in the $6 \mathrm{~h}$ of profiling with batch $\mathrm{D}$ tablets giving the highest drug release of $91 \%$. Apart from the Batch $\mathrm{G}$ tablets, all the other tablets released over $70 \%$ of their drug content within the $6 \mathrm{~h}$ of dissolution testing. These results show that with the inclusion of PEG (Mol. Wt. 4000 and 6000) in the formulations, drug release increased when compared to the formulations containing carnauba wax alone. However, the drug release increased only to the extent of carnauba wax concentration as evident with all the batches of tablets containing $50 \mathrm{mg}(12.20 \% \mathrm{w} / \mathrm{w})$ of carnauba wax showing percentage drug release ranging from 71 to $91 \%$ while those containing $100 \mathrm{mg}(24.40 \% \mathrm{w} / \mathrm{w})$ of carnauba wax released a percentage range of 60.5 to $87 \%$. As the concentration of carnauba wax increased, the drug release decreased. Also, all the batches formulated with PEG exhibited a percentage drug release of over $80 \%$ in $6 \mathrm{~h}$ irrespective of the amount of carnauba wax indicating that PEG increased the release of the drug. PEG as a hydrophilic polymer may be acting as a channeling agent for the drug by increasing the porosity of the tablet matrix when in contact with the dissolution fluid and forming channels for the drug to diffuse out of the matrix of the tablets. However, a 1:1 combination of PEG 4000 and 6000 produced the highest release of $91 \%$ in formulation prepared with $50 \mathrm{mg}(12.20 \% \mathrm{w} / \mathrm{w})$ of carnauba wax (Batch D) as against the 1:2 combination of PEG 4000 and 6000 in Batch K tablets prepared with $100 \mathrm{mg}(24.40 \% \mathrm{w} / \mathrm{w})$ of carnauba wax with a maximum drug release of 83 $\%$. In effect, the combination of the PEGs produced maximum modulatory release of the drug from the tablets, but it was influenced by the concentration of carnauba wax forming the matrix of the tablets. The results of the release mechanism and kinetics of the drugs from the matrix tablets are shown in Table 4 which showed that the drug release was most consistent with the Korsmeyer-Peppas model since the correlation coefficient $\left(r^{2}\right)$ values were $\geq 0.95$ and with the release exponent $(n)>0.89$, indicates that drug release was Super Case-II diffusion mediated (Eichie et al., 2008; Eraga and Iwuagwu, 2014). 


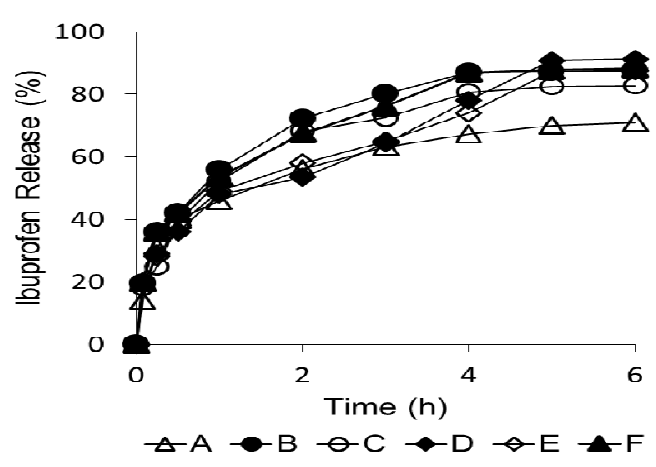

Fig 3: Dissolution profile of ibuprofen matrix tablets prepared with $12.20 \% \mathrm{w} / \mathrm{w}(50 \mathrm{mg})$ carnauba wax (A-F)

Conclusion; Sustained release matrix tablets can be prepared using hydrophobic carriers which act as very good drug retardant. PEG can be used as a channeling agent in waxy matrix to regulate the release of drug. Carnauba wax hence acts as a good retardant to the release of drug. With the addition of PEG that acted as a channeling agent, the drug release increased with over $80 \%$ in $6 \mathrm{~h}$.

\section{REFERENCES}

Ahmed, MK; Khalil, YI (2014). Formulation and in vitro evaluation of diclofenac sodium sustained release matrix tablets using carnauba wax as a matrix former. Int. J. Pharm. Pharm. Sci. 6(2): 461-466.

Arhewoh, MI; Eraga, SO; Avbunudiogba, JA; Fregene, Q; Okah, P (2015). Preparation and evaluation of matrix tablets of metronidazole using carnauba wax. Bri. J. Pharm. Res. 8(5): 18.

Avbunudiogba, JA; Cash-Torunarigha, OE; Onah, I (2013). Effect of humidity on the physical properties of aspirin tablets produced by melt granulation and slugging. IOSR J. Pharm. Bio. Sci. 7(5): 20-25.

British Pharmacopoeia (2009). Vol. III. The Pharmaceutical Press, Her Majesty's Stationer Office, London. pp. 6578-6585.

Bruschi, ML (2015). Strategies to Modify the Drug Release from Pharmaceutical Systems. Woodhead Publishing Limited, Cambridge, pp. 93-97.

Carstensen, JJ; Chan, PC (1977). Flow rates and repose angles of wet processed granulations. $J$. Pharm. Sci. 60: 1235-1239.

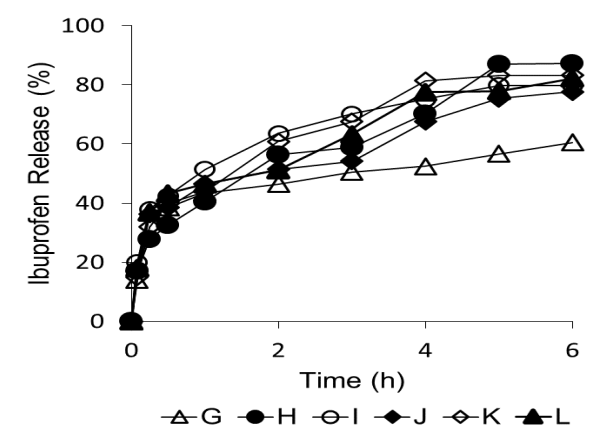

Fig 4: Dissolution profile of ibuprofen matrix tablets prepared with $24.40 \%$ w/w (100 mg) carnauba wax (G-L)

Eichie, FE; Okor, RS; Esi, O (2008). Matrix release from tablets prepared with aqueous dispersion of an acrylate methacrylate (a water insoluble) copolymer as binder. Int. J. Health Res. 1(4): 235-240.

Eraga, SO; Erebor, JO; Iwuagwu, MA (2014). Preliminary investigation into the use of lima bean (Phaseolus lunatus) starch as a tablet disintegrant. J. Pharm. Allied Sci. 11(2): 20812091.

Higuchi, T (1968). Mechanism of sustained action medication. Theoretical analysis of rate release of solid drugs dispersed in solid matrices. $J$. Pharm. Sci. 52: 1145-1149.

Ibn-Razzak, MSM; Khan, F; Hossain, M; Khan, MZR; Azad, MAK; Reza, MS (2009). Effects of channeling agents on the release pattern of theophylline from kollidon SR based matrix tablets. Pak. J. Pharm. Sci. 22(3): 303-307.

Iwuagwu, MA; Nwaoshai, CA; Iseghohi, FE (1986). Investigations into the binding and disintegrants properties of starches from selected dioscoreaceae plants. Nig. J. Pharm. Sci. 2: 1022.

Korsmeyer, RW; Gurny, R; Doelker, E; Buri, P; Peppas, NA (1983). Mechanisms of solute release from porous hydrophilic polymers. Int. $J$. Pharm. 15: 25-35.

Maheshwari, M; Ketkar, AR; Chauhan, B; Patil, VB; Paradkar, AR (2003). Preparation and characterization of ibuprofen-cetyl alcohol beads by melt solidification technique: effect of variables. Int J Pharm 261: 57-67. 
Mehta, A; Barker, CG (1994). Disorder, memory and avalanches in sand piles. Europhys. Lett. 27(7): 501-506.

Onyechi, JO; Okafo, SE (2016). Evaluation of carnauba wax in sustained release diclofenac sodium tablet formulation. J. Chem. Pharm. Res. 8(3):714-721.

Oyi, AR; Allagh, TS; Olayemi, OJ (2009). Comparative binding effects of wheat, rice and maize starches in chloroquine phosphate tablet formulations. Res. J. Appl. Sci. Eng. Technol. 1: 77-80.

Paradkar, AR; Ambike, AA; Jadhav, BK; Mahadik, KR (2004). Characterization of curcumin-PVP solid dispersion obtained by spray drying. Int. $J$. Pharm. 271: 281-286.

Richards, JH (1972). Powder flow and compaction. In: Carter, SJ (ed) Tutorial Pharmacy. Pitman Medical Publishing Ltd., London, pp.211-233.

Robson, HJ; Craig, DQM; Deutsch, D (1999). An investigation into the release of cefuroxime axetil from taste masked stearic acid microspheres. Part
1: The influence of the dissolution medium on the drug release profile and the physical integrity of the microspheres. Int. J. Pharm. 190: 183-192.

Rudnic, EM; Schwartz, JB (2006). Oral solid dosage forms. In: Troy, DB; Beringer, $\mathrm{P}$ (eds) Remington - The Science and Practice of Pharmacy, Lippincott Williams and Wilkins, Baltimore, pp. 889-928.

Uddin, MB; Chowdhury, JA; Azam, KR; Jalil, R; Reza, MS (2009). Preparation and evaluation of in vitro release kinetics of theophylline loaded matrix tablet based on Eudragit NE $30 \mathrm{D}$ and Eudragit RS 30 D. Dhaka Univ. J. Pharm. Sci. 8(2): 153-159.

Veronese, FM; Pasut, G (2005). PEGylation, successful approach to drug delivery. Drug Discov. Today. 10(21): 1451-1458.

Wagner, JG (1969). Interpretation of percent dissolved-time plots derived from in vitro testing of conventional tablets and capsules. J. Pharm. Sci. 58(10): 1253-1257. 